\title{
Eine Glucose-Dehydrogenase für die Glucose-Bestimmung in Körperflüssigkeiten ${ }^{1}$ )
}

\author{
Von D. Banauch, W. Brümmer, W. Ebeling, H. Metz, H. Rindfrey und H. Lang, \\ Biochemische Forschung, E. Merck Darmstadt,
}

\author{
K. Leybold, \\ Zentrallaboratorium, Städtisches Krankenhaus Kiel und
}

W. Rick, Institut für Klinische Chemie und Laboratoriumsdiagnostik, Universität Düsseldorf

(Eingegangen am 30. August 1974/23. Dezember 1974)

Herrn Professor Dr. H. J. Staudinger zum 60. Geburtstag gewidmet

\begin{abstract}
Die Isolierung der Glucose-Dehydrogenase aus Bacillus megaterium M 1286 wird beschrieben. Daten über die Spezifität des Enzyms gegenüber Kohlenhydraten werden angegeben. Mit Hilfe dieses Enzyms ist eine spezifische Glucose-Bestimmung in Körperflüssigkeiten möglich. Durchführung und Ergebnisse von vier verschiedenen Varianten der Glucose-Bestimmung mit Glucose-Dehydrogenase werden dargestellt: Endpunkt-Bestimmung im ultravioletten Bereich (UV-Methode), Bestimmung mit Formazan als Endprodukt (Farbmethode), kinetische Bestimmung im ultravioletten Bereich (kinetische Methode) und kontinuierliche Analyse im ultravioletten Bereich (AutoAnalyzer-Methode).
\end{abstract}

\section{A glucose dehydrogenase for the determination of glucose concentrations in body fluids}

The isolation of glucose dehydrogenase from Bacillus megaterium M 1286 is outlined. Data on the specificity of the enzyme towards carbohydrates are given. A specific method for glucose determination using this enzyme was developed. Methods and results of four variants of this glucose determination are presented: End point determination in the UV range, determination with formazan as reaction product, kinetic determination in the UV range, and continuous flow analysis in the UV range (AutoAnalyzer method).

Glucose-Dehydrogenase katalysiert die Dehydrierung von $D$-Glucose zu $D$-Gluconolacton, wobei der Wasserstoff auf NAD oder NADP übertragen wird. Das Vorkommen des Enzyms in Säugetierleber (1) und Bakteriensporen (2) ist schon seit längerer Zeit bekannt. Bisher wurden jedoch weder Reindarstellung noch Verwendung als analy tisches Hilfsmittel beschrieben. Störend für den analytischen Einsatz sind die NADH-verbrauchenden Enzyme (sog. „NADH-Oxidasen“), die GlucoseDehydrogenase bei der Aufarbeitung hartnäckig begleiten (3).

Wir beschreiben die Herstellung von.praktisch NADHOxidase-freier bakterieller Glucose-Dehydrogenase und verschiedene Varianten einer spezifischen Bestimmung von Glucose in Körperflüssigkeiten mit Hilfe dieses Enzyms ${ }^{2}$ ).

\footnotetext{
1) Sonderdruck-Anforderungen Dr. H. Lang an: $\quad$ Biochemische Forschung E. Merck Requests for reprints to: D-61 Darmstadt, Postfach 4119

2) Enzyme Glucose- $\quad=\beta-D$-Glucose: NAD-oxidoreductase, Dehydrogenase EC 1.1.1.47

Mutarotase = Aldose-1-epimerase, EC 5.1.3.3

Diaphorase = NADH: lipoamide oxidoreductase, EC 1.6.4.3
}

\section{Material und Methoden}

\section{Meßgeräte}

Photometrische Messungen mit Eppendorf-Photometer $1101 \mathrm{M}$ mit Kompensationsschreiber 6511 . Meßtemperatur $25^{\circ} \mathrm{C}$.

\section{Reagenzien}

Glucose-6-phosphat Dinatriumsalz von Boehringer/Mannheim, 3-(4,5-Dimethyl-2-thiazolyl)-2,5-diphenyltetrazolium-bromid von EGA/Steinheim. Für die Richtigkeitsuntersuchung StandardGlucose des National Bureau of Standards (NBS, Washington). Alle anderen Reagenzien von E. Merck/Darmstadt (Reinheitsgrad p. A. oder für biochemische Zwecke). Für die Spezifitätsuntersuchungen verwendete Kohlenhydrate waren dünnschichtchromatographisch auf Abwesenheit von Glucose geprüft.

\section{Gewinnung der Bakterienmasse}

Glucose-Dehydrogenase wird aus Kulturen des Stammes Bacillus megaterium M 1286 gewonnen. Die Anzucht erfolgt in Fermentern unter aeroben Bedingungen bei $28^{\circ} \mathrm{C}$ in einer Nährlösung, die neben Mineralsalzen Hefeextrakt, Pepton und Glucose enthält. Meßbare Glucose-Dehydrogenase-Aktivität tritt erstmals gegen Ende der logarithmischen Wachstumsphase auf und erreicht nach 20 bis 24 Stunden Kulturdauer ihr Maximum. Das Enzym ist intrazellulär lokalisiert und wird nicht in das Medium ausgeschieden. Die Bakterienmasse wird mit einem Schlammseparator abgetrennt und anschließend mit einem Manton-Gaulin-Hochdruckhomogenisator aufgeschlossen. 
Tab. 1. Isolierung der Glucose-Dehydrogenase, Verlauf.

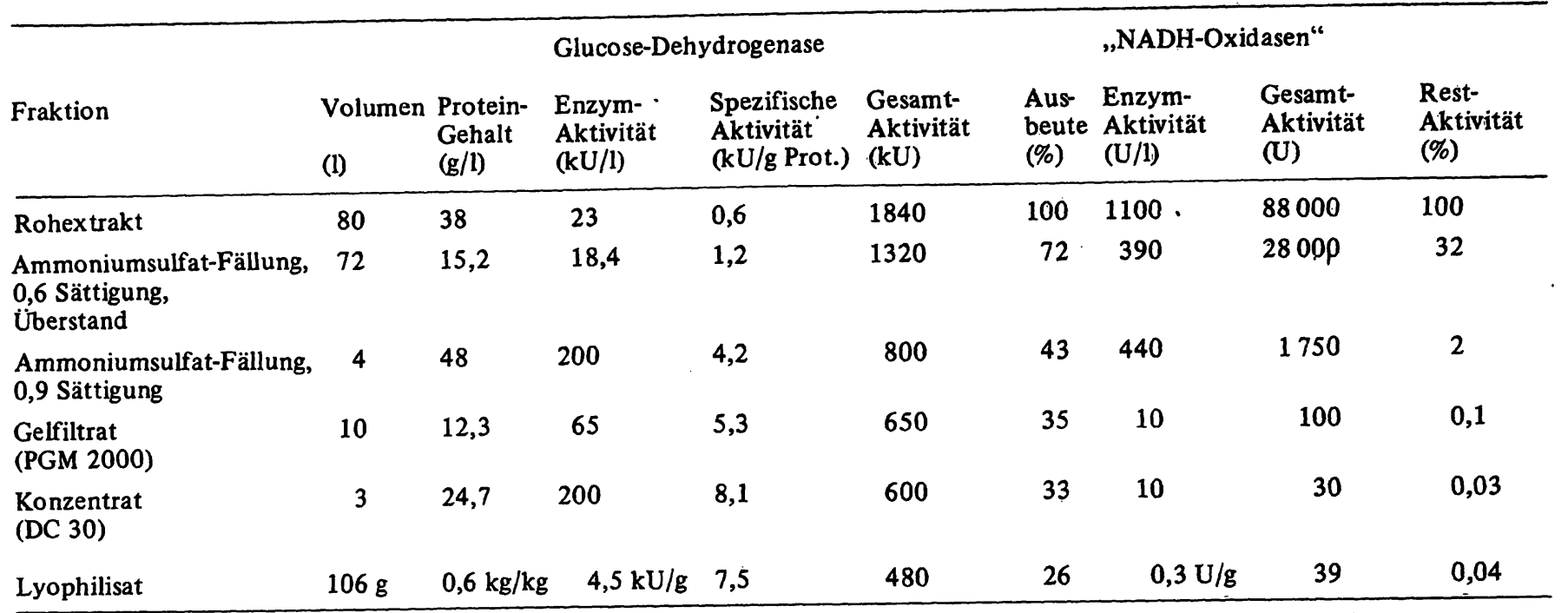

\section{Isolierung der Glucose-Dehydrogenase}

5 bis 501 aufgeschlossener Bakterienschlamm, Glucose-Dehydrogenase-Aktivität etwa $20 \mathrm{kU} / \mathrm{l}$, werden 2 Stunden bei $2500 \mathrm{~g}$ zentrifugiert. Der Rückstand (Bakterientrümmer) wird verworfen und der Überstand $(=$ Rohextrakt) mit Ammoniumsulfat fraktioniert. Die enzymhaltige Proteinfraktion fällt bei einer Ammoniumsulfat-Sättigung zwischen 0,6 und 0,9 aus. Sie wird abzentrifugiert und in Wasser zu $1 / 20$ des Ausgangsvolumens $(0,25$ bis $2,51)$ gelöst. Die Lösung wird zur Abtrennung niedermolekularer Verunreinigungen (insbesondere von Farbstoffen) an Merckogel PGM 2000 (= Polyäthylenglycol-dimethacrylat) chromatographiert. Die Säule ist mit 0,25 mol/1 Tris/HCl-Puffer, pH 6,8, äquilibriert. Das Eluat wird durch Ultrafiltration (Amicon-HollowFiber-Gerät; Modell DC 30) bis zu einer Leitfähigkeit von etwa $1 \mathrm{mS}$ gegen Wasser dialysiert und danach auf eine Aktivität von etwa $200 \mathrm{kU} / 1$ Lösung konzentriert. Das Enzym-Konzentrat wird gefriergetrocknet; das Lyophilisat hat eine durchschnittliche Aktivität von $3 \mathrm{kU} / \mathrm{g}$ Trockensubstanz. Die Ausbeute beträgt $25 \%$. Der Restgehalt an NADH-Oxidase beträgt $0,01 \%$ (bezogen auf die Glucose-Dehydrogenase-Aktivität).

Tabelle 1 zeigt den Verlauf der Enzymisolienung und die Abreicherung der NADH-Oxidase-Aktivität. Über die Darstellung und Charakterisierung des Reinstenzyms wird von Pauly und Pfleiderer (4) an anderer Stelle berichtet.

Enzy maktivitäts-Bestim mungen

Bestimmung der Aktivität von Glucose-DehydrogenasePräparaten

$3,00 \mathrm{ml} 100 \mathrm{mmol} / 1$ Trispuffer $\mathrm{pH} \mathrm{7,5,} \mathrm{enthaltend}$ $168 \mathrm{mmol} / 1 \mathrm{D}$-Glucose im Mutarotationsgleichgewicht

$0,10 \mathrm{ml}$ Probelösung (entsprechend etwa 0,1 U GlucoseDehydrogenase)

$0,05 \mathrm{ml}$ NAD-Lösung ( $90 \mathrm{mmol} / 1$ in bidest. Wasser)

mischen und bei $365 \mathrm{~nm} \Delta \mathrm{E} / \mathrm{min}$ bestimmen $(\mathrm{d}=1 \mathrm{~cm})$. Berechnung der Glucose-Dehydrogenase-Aktivität: $\mathrm{kU} / 1$ Enzymlösung $=\Delta E_{365} / \min \times 9,26$.

\section{Bestimmung der Aktivität von „NADH-Oxidase“"}

$3,00 \mathrm{ml} \quad 100 \mathrm{mmol} / 1$ Trispuffer $\mathrm{pH} \mathrm{7,5}$

$0,10 \mathrm{ml}$ Probelösung

$0,05 \mathrm{ml}$ NADH-Lösung ( $11 \mathrm{mmol} / 1$ in bidest. Wasser)

mischen und bei $365 \Delta \mathrm{E} / \mathrm{min}$ bestimmen $(\mathrm{d}=1 \mathrm{~cm})$. Berechnung der NADH-Oxidase-Aktivität: $\mathrm{kU} / 1$ Enzymlösung $=\Delta E_{365} / \min \times 9,26$.

\section{Spezifität der Glucose-Dehydrogenase}

Bakterielle Glucose-Dehydrögenase wurde von Sadoff (5) als absolut spezifisch für Glucose beschrieben. Andere Autoren fanden geringe Nebenaktivitäten gegen Mannose (6), Xylose (7-10), Galaktose (7, 9) und Arabinose (8). Das von uns untersuchte Enzym reagiert mit mehreren Monosacchariden. In Tabelle 2 ist die relative Umsatzgeschwindigkeit verschiedener Zucker bei Anwesenheit von Glucose-Dehydrogenase dargestellt: außer Glucose $(100 \%)$ reagieren mit Reaktionsraten von $\geqslant 1 \%$ :

2-Desoxyglucose (125\%), 2-Amino-2-desoxyglucose (Glucosamin) (31\%), D-Xylose (15\%), D-Mannose (8\%), 6-Amino-6-desoxyglucose $(6 \%)$ und Cellobiose (1\%).

Reaktionsgeschwindigkeiten zwischen 1 und $0,1 \%$ des Glucose-Umsatzes zeigen $D$-Ribose, 2-Desoxyribose und Lactose. Diese Aktivitäten sind analytisch noch sicher

Tab. 2. Spezifität von Glucose-Dehydrogenase gegenüber Kohlenhydraten.

Aktivität gegenüber Glucose $=100$. Die Tabelle enthält die Substanzen mit Aktivitäten $\geqslant 0,1$. Einzelheiten siehe Text.

Testansatz: $160 \mathrm{mmol} / \mathrm{l}$ Kohlenhydrat; $1,4 \mathrm{mmol} / 1 \mathrm{NAD}$; $0,63 \mathrm{mmol} / 1$ Trispuffer, pH 7,6. Messung der Extinktionsänderung bei $365 \mathrm{~nm}$ während 3 Minuten. Einzelheiten siehe Text.

\begin{tabular}{lc}
\hline Substrat & Aktivität \\
\hline 2-Desoxyglucose & 125 \\
$\beta$-D-Glucose & 100 \\
$D$-Glucosamin & 31 \\
$D$-Xylose & 15 \\
$D$-Mannose & 8 \\
6-Amino-6-desoxyglucose & 6 \\
Cellobiose & 1 \\
D-Ribose & 0,8 \\
Lactose & 0,7 \\
2-Desoxyribose & 0,1 \\
\hline
\end{tabular}


nachweisbar, eine Störung der Glucose-Bestimmung tritt wegen der sehr geringen Umsatzgeschwindigkeiten dieser Substanzen jedoch nicht auf.

Praktisch keine Reaktion ( 0,03 bis $0 \%$ des GlucoseUmsatzes) ergeben folgende Verbindungen: Galaktose, 2-Desoxygalaktose, Fructose, Talose, Thioglucose, Glucose-1-phosphat, Glucose-6-phosphat, 2-(N-Acetyl-) glucosamin, 2-(N-Methyl-)glucosamin, 2-(N-Benzyl-) glucosamin, Arabinose, Gluconsäure- $\delta$-lacton, Glucuronsäure- $\gamma$-lacton, Trehalose, Saccharose. Die gleiche Spezifität erhält man bei Verwendung von NADP als Coenzym.

Von den in Tabelle 2 aufgeführten Substanzen können von der Umsatzgeschwindigkeit her 2-Desoxyglucose, Glucosamin, Xylose, Mannose, 6-Amino-6-desoxyglucose und Cellobiose die Glucose-Bestimmung mit Glucose-Dehydrogenase beeinflussen. Eine Störung der Glucose-Bestimmung in Körperflüssigkeiten ist jedoch nur in Ausnahmefällen möglich:

2-Desoxyglucose, 6-Amino-6-desoxyglucose und Cellobiose kommen im Säugetierorganismus nicht vor. Nicht acetyliertes Glucosamin tritt nur in Mucoproteiden und Mucopolysacchariden gebunden auf (11) und wird in dieser Form von Glucose-Dehydrogenase nicht umgesetzt. Die Angaben über das Vorkommen von Xylose in Körperflüssigkeiten sind unterschiedlich: während mehrere Autoren $(12,13,14)$ keine Xylose oder nur Spuren finden, berichtet Hawkins (15) über Xylose-Konzentrationen im Blut von durchschnittlich $167 \mu \mathrm{mol} / \mathrm{l}$ $(2,5 \mathrm{mg} / 100 \mathrm{ml})$. Modellversuche der Methode mit Xylose-Zusatz ergeben, daß eine Störung der GlucoseBestimmung erst ab $2 \mathrm{mmol} / \mathrm{l}(30 \mathrm{mg} / 100 \mathrm{ml})$ Xylose meßbar ist. Bei oraler Xylose-Belastung treten Konzentrationen von mehr als $4,66 \mathrm{mmol} / 1(70 \mathrm{mg} / 100 \mathrm{ml})$ auf (16); in diesem Fall ist die Blutzucker-Bestimmung mit Glucose-Dehydrogenase nicht möglich. Der Normalwert für Mannose liegt bei etwa 55,5 $\mu \mathrm{mol} / 1(1 \mathrm{mg} /$ $100 \mathrm{ml}$ ) (17). Mannose reagiert mit Glucose-Dehydrogenase noch langsamer als Xylose, so daß auch bei zwanzigfacher Überschreitung des Normalwertes keine Störung der Glucose-Bestimmung erfolgt.

\section{Glucose-Bestimmung in Körperflüssigkeiten}

Endpunktbestimmung im ultravioletten Bereich (UV-Methode)

\section{Bestimmungsansatz}

Glucose-Dehydrogenase ist spezifisch für $\beta$ - $D$-Glucose (18). Damit nach Abreagieren der $\beta$-Form die langsame Eigen-Mutarotation nicht der geschwindigkeitsbestimmende Schritt der Gesamtreaktion wird, muß das Testsystem Mutarotase enthalten. Dieses Enzym wird aus Rindernieren nach Fishman et al. $(19,20)$ gewonnen.

Zur Glucose-Bestimmung werden die beiden Enzyme in $120 \mathrm{mmol} / \mathrm{l}$ Phosphatpuffer pH 7,6 gelöst, der Test- ansatz enthält pro $\mathrm{ml} 9 \mathrm{U}$ Glucose-Dehydrogerase und 0,3 U Mutarotase. Nach Zugabe der Probe (mit Perchlorsäure enteiweißtes Vollblut, Plasma oder Serum) wird die Reaktion mit NAD-Konzentrat gestartet (Endkonzentration im Test: 2,0 mmol/1 NAD).

\section{Arbeitsvorschriften für die UV-Methode}

\section{Einzelbestimmung}

In einer Küvette $(\mathrm{d}=1 \mathrm{~cm})$ mischen:

Puffer-Enzym-Lösung

$2,00 \mathrm{ml}$

Probelösung

$0,20 \mathrm{ml}$

Extinktion registrieren, dann Reaktion starten mit

NAD-Lösung

$0,02 \mathrm{ml}$

Nach Ablauf der Reaktion (5 Minuten) Endextinktion registrieren. Meßwellenlänge 334,340 oder $365 \mathrm{~nm}$. Extinktion des Startreagenzes durch Reagenzienblindwert berücksichtigen.

Die Berechnung des Glucose-Gehaltes erfolgt mit Hilfe des für die Meßwellenlänge gültigen molaren Extinktionskoeffizienten von NADH.

\section{Serienbestimmung}

Reagenziengemisch: 100 Volumenteile Puffer-EnzymLösung mit einem Volumenteil NAD-Lösung mischen.

In Reagenzgläser pipettieren:

$\begin{array}{lll} & \text { Analyse } & \text { Probenleerwert } \\ \text { Reagenziengemisch } & 1,00 \mathrm{ml} & - \\ \text { Puffer-Enzym-Lösung } & - & 1,00 \mathrm{ml} \\ \text { Probelösung } & 0,10 \mathrm{ml} & 0,10 \mathrm{ml}\end{array}$

5 Minuten bei Raumtemperatur stehenlassen, dann die Extinktion der Analyse gegen den Probenleerwert bei 334, 340 oder $365 \mathrm{~nm}$ messen. Dabei muß ein Küvettenfehler ausgeschlossen sein. Für jede Serie einen NAD-Blindwert bestimmen (statt Probelösung Enteiweißungsmittel einsetzen).

Die Berechnung erfolgt wie bei der Einzelbestimmung. In Abbildung 1 ist in Kurve 1 der Glucose-Umsatz bei Einsatz des Enzymgemisches Glucose-Dehydrogenase/

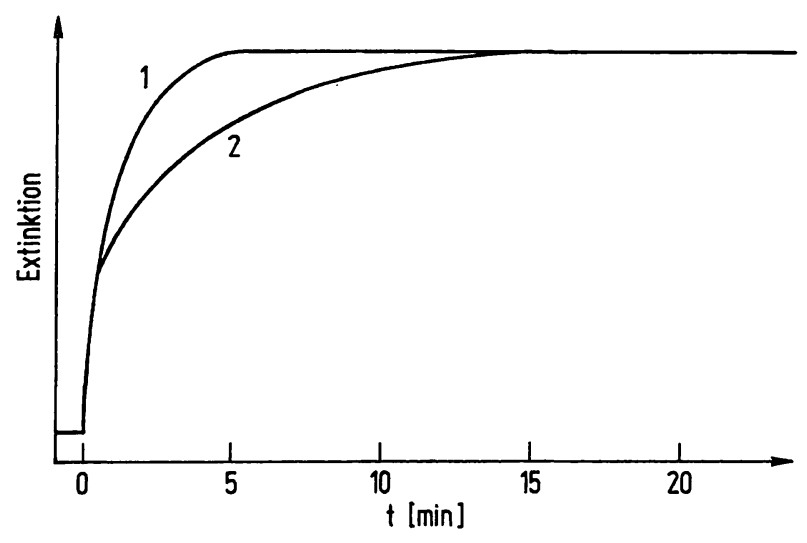

Abb. 1. Glucose-Bestimmung mit Glucose-Dehydrogenase Zeit-Umsatz-Kurve der Endpunkt-Bestimmung im UVBereich (UV-Methode)

1 mit Mutarotase, 2 ohne Mutarotase 
Mutarotase dargestellt. Die Enzymaktivitäten im Test sind so bemessen, daß auch bei hohen Glucose-Konzentrationen die Reaktion nach fünf Minuten abgeschlossen ist. Bei Verwendung von Enzympräparaten mit $\leqslant 0,01 \%$ NADH-Oxidase ist die Endextinktion mehr als $30^{\circ}$ Minuten konstant. Kurve 2 zeigt die Umsatzkurve ohne Mutarotase: nach Abreagieren der $\beta$-Glucose wird die Mutarotation der geschwindigkeitsbestimmende Schritt, so daß die Extinktion erst nach etwa 15 Minuten einen konstanten Wert erreicht.

\section{Linearität und Richtigkeit der UV-Methode}

Die Richtigkeit der Methode wurde durch Analyse von wäßrigen Glucose-Lösungen (NBS Standard-Referenzglucose) bekannten Gehaltes überprüft. Abbildung 2 zeigt, daß bis zu Konzentrationen von $55,5 \mathrm{mmol} / 1$ $(1000 \mathrm{mg} / 100 \mathrm{ml})$ Glucose die theoretische Menge wieder gefunden wird. Die korrekte Wiederfindung im Serum zeigt Tabelle 3. Berechnung des Glucose-Gehaltes mit dem korrigierten Extinktionskoeffizienten für $\mathrm{NADH}, \epsilon_{365}=3,4 \mathrm{~cm}^{2} / \mu \mathrm{mol}(21)$.

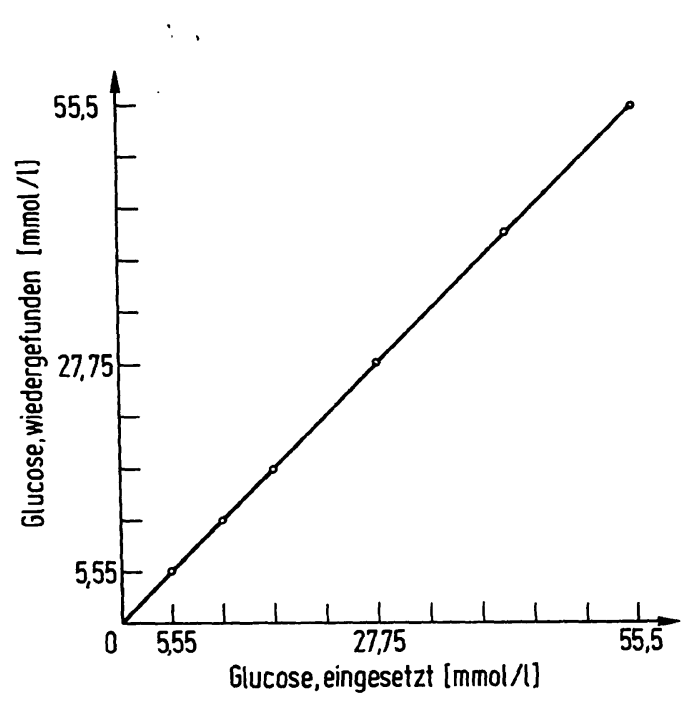

Abb. 2. Glucose-Bestimmung mit Glucose-Dehydrogenase Endpunkt-Bestimmung im UV-Bereich (UV-Methode) Prüfung der Richtigkeit mit wäßrigen Lösungen von NBS-Standard-Glucose. Ansatz siehe Text. $55,5 \mathrm{mmol} / 1 \mathrm{Glucose}=1000 \mathrm{mg} / 100 \mathrm{ml}$

Tab. 3. Glucose-Bestimmung mit Glucose-Dehydrogenase. Wiederfindung mit der Endpunkt-Bestimmung im ultravioletten Bereich (UV-Methode).

Material: NBS-Standard-Glucose.

Wiederfindung: Mittelwerte aus 5-fach-Bestimmungen. Ansatz siehe Text.

\begin{tabular}{llll}
\hline $\begin{array}{l}\text { Zugesetzte Glucose } \\
\text { mmol/1 (mg/100 ml Serum) }\end{array}$ & $\begin{array}{l}\text { Wiederfindung } \\
\mathrm{mmol} / 1(\mathrm{mg} / 100 \mathrm{ml})\end{array}$ & $\begin{array}{l}\text { VK } \\
(\%)\end{array}$ \\
\hline 11,1 & $(200)$ & $11,06(199,3)$ & 1,44 \\
$22,2 \quad(400)$ & $22,02(396,8)$ & 0,90 \\
$44,4 \quad(800)$ & $44,17(796,0)$ & 0,57 \\
\hline
\end{tabular}

Bestimmung mit Formazan als Endprodukt (Farbmethode)

Die Glucose-Bestimmung mit Glucose-Dehydrogenase kann auch mit Hilfe einer Farbreaktion erfolgen. Man überträgt dabei den Wasserstoff durch eine Diaphorase von NADH auf das Tetrazoliumsalz 3-(4,5-Dime thyl-2thiazolyl)-2,5-diphenyltetrazolium-bromid, wobei ein tiefblaues Formazan entsteht. Die Methode ist einfach in der Ausfuihrung (ein Reagenzien- oder'Probenblindwert muß nicht berücksichtigt werden), etwa fünfmal empfindlicher als die UV-Methode und benötigt kein UV-Photometer.

Endkonzentrationen im Testansatz: $60 \mathrm{mmol} / 1$ Phosphatpuffer pH 7,0 (enthält pro ml 5 U Glucose-Dehydrogenase, 0,2 U Mutarotase, 0,2 U Diaphorase (Clostridium kluyveri)), 1,25 mmol/1 NAD, 1,2 mmol/1 3-(4,5-Dimethyl-2-thiazolyl)-2,5-diphenyltetrazolium-bromid und $0,2 \mathrm{mmol} / 1 \mathrm{~N}-\mathrm{A}$ thyl-maleinimid. Enteiweißung mit Trichloressigsäure.

\section{Arbeitsvorschrift für die Farbmethode}

In einem Reagenzglas mischen

Reagenzlösung

$2,00 \mathrm{ml}$

Probelösung

$0,05 \mathrm{ml}$

30 Minuten bei Raumtemperatur stehenlassen (direktes Sonnenlicht vermeiden), dann innerhalb von weiteren 30 Minuten die Extinktionen von Analyse und Reagenzlösung gegen Wasser messen. Die Differenz der Extinktionen geht in die Berechnung ein (Wellenlänge zwischen 540 und $600 \mathrm{~nm}$ ). Für jede Analysenserie muß ein Glucose-Standard bestimmt werden. Das Ergebnis der Standard-Bestimmung wird der Berechnung zugrundegelegt.

Die Bezugskurve für den Farbtest ist bis $27,75 \mathrm{mmol} / 1$ $(500 \mathrm{mg} / 100 \mathrm{ml})$ Glucose linear. Weitere Ergebnisse dieses Tests siehe die folgenden Abschnitte.

Kinetische Bestimmung im ultravioletten Bereich (kinetische Methode)

Zur Glucose-Bestimmung mit Glucose-Dehydrogenase . ist außer den beschriebenen Endpunkt-Methoden auch das kinetische Verfahren geeignet. Durch Variation des Verhältnisses von Enzymaktivität zur Probenmenge im Testansatz kann die Reaktionsgeschwindigkeit und damit die Dauer des linearen Teils des Reaktionsverlaufes innerhalb weiter Grenzen gewählt werden. Einsatz einer hohen Enzymak tivität (im Vergleich zur Probenmenge) bewirkt eine große Meßempfindlichkeit bei kurzer Dauer des linearen Reaktionsverlaufes; dieser Ansatz ist für die mechanisierte Durchführung der kinetischen Methode geeignet (22). Einsatz einer niedrigen Enzymaktivität (im Vergleich zur Probenmenge) bewirkt eine geringere Meßempfindlichkeit bei langer Dauer des linearen Reaktionsverlaufes; dieser Ansatz ist für die manuelle Durchführung der Methode geeignet. Die kinetische Methode kann mit und ohne Enteiweißung der Probe durchgefuhrt werden. 
Als Beispiel wird anschließend eine manuelle Variante der kinetischen Methode mit Enteiweißung und einer Meßzeit von 3 Minuten beschrieben. Ein Vorteil dieser Methode ist der sehr geringe Bedarf an Glucose-Dehydrogenase, Mutarotase wird nicht benötigt. Bei Einsatz von Perchlorsäure-enteiweißten Proben ist die Bezugskurve bis $55 ; 5 \mathrm{mmol} / 1(1000 \mathrm{mg} / 100 \mathrm{ml})$ linear. Die Testlösung (160 mmol/1 Tris-Puffer $\mathrm{pH} 7,8)$ enthält $0,6 \mathrm{U} / \mathrm{ml} \mathrm{Glu-}$ cose-Dehydrogenase und 2,1 mmol/1 NAD.

\section{Arbeitsvorschrift für die kinetische Methode Beispiel mit Enteiweißung und Meßzeit von 3 Minuten}

Reagenzlösung, Glucose-Standard und enteiweiß te Probe auf $25^{\circ} \mathrm{C}$ temperieren. Der Küvettenhalter muß auf $25^{\circ} \mathrm{C}$ thermostatisiert sein. In eine Küvette $(\mathrm{d}=1 \mathrm{~cm})$ pipettieren:

\section{Reagenzlösung}

$2,00 \mathrm{ml}$

Probelösung $0,50 \mathrm{ml}$

mischen, nach einer Wartezeit von etwa 30 Sekunden drei Minuten lang die Extinktionsänderung bei 334, 340 oder 365 nm messen, Extinktionsänderung pro Minute berechnen. Für jede Analysenserie muß ein GlucoseStandard bestimmt werden. Das Ergebnis der StandardBestimmung wird der Berechnung zugrundegelegt.

Ergebnisse dieses Tests siehe die folgenden Abschnitte.

\section{Kontinuierliche Analyse im ultravioletten} Bereich (AutoAnalyzer-Methode)

Die Glucose-Bestimmung mit Glucose-Dehydrogenase in continuous-flow-Technik erfolgt am AutoAnalyzer II. Abbildung 3 zeigt das Fließbild. Die etwa 1:5 mit $\mathrm{H}_{2} \mathrm{O}$-Brij verdünnte Serumprobe wird in einem 3-ZollDialysator gegen das Reagenz dialysiert; die Reaktion erfolgt in einer Mischspirale von 80 Windungen bei Raumtemperatur. Das gebildete NADH wird bei $340 \mathrm{~nm}$ gemessen. Die Reagenzlösung enthält die gleichen Bestandteile und Konzentrationen wie für die UV-Endpunkt-Methode in Serienanalyse. Eine Methode mit geringerem Enzym-Bedarf ist in Entwicklung.

Es wird mit einer Probenfrequenz von 60 pro Stunde gearbeitet, wobei die Analysendauer für die einzelne
Probe etwa 10 Minuten beträgt. Wenn eine $5,55 \mathrm{mmol} / \mathrm{l}$ $(100 \mathrm{mg} / 100 \mathrm{ml})$-Probe direkt auf eine $27,75 \mathrm{mmol} / 1$ $(500 \mathrm{mg} / 100 \mathrm{ml})-$ Probe folgt, wird diese Probe um $5 \%$ zu hoch gefunden. Die Bezugskurve ist bis $27,75 \mathrm{mmol} / 1$ $(500 \mathrm{mg} / 100 \mathrm{ml})$ linear.

\section{Bewertung der Varianten der Glucose-Bestimmung mit Glucose-Dehydrogenase}

In Tabelle 4 werden die vier Methoden der GlucoseBestimmung mit Glucose-Dehydrogenase verglichen. Bei der Farbmethode und der AutoAnalyzer-Methode wird der effektive Zeitbedarf pro Analyse durch versetztes Arbeiten in der Serie stark verringert.

\section{Störungen der Methoden}

Bei den Methoden im ultravioletten Bereich werden durch die einstufige Reaktion unspezifische Nebenreaktionen vermieden. Diese Methoden sind daher neben der Blutzuckerbestimmung besonders für die Glucose-Bestimmung im Urin geeignet. Bei der Farbmethode wirken Reduktionsmittel durch zusätzliche Formazanbildung störend; der Einfluß von SH-Verbindungen (Glutathion) wird jedoch durch den Zusatz von N-Äthylmaleinimid unterdrückt.

Bei der Blutzucker-Bestimmung erfolgt bei allen beschriebenen Methoden-Varianten keine Beeinflussung der Meßergebnisse durch folgende Substanzen: Harnsäure $595 \mu \mathrm{mol} / 1(10 \mathrm{mg} / 100 \mathrm{ml})$, Bilirubin $342 \mu \mathrm{mol} / 1$ $(20 \mathrm{mg} / 100 \mathrm{ml})$, Glutathion $651 \mu \mathrm{mol} / 1(20 \mathrm{mg} / 100 \mathrm{ml})$, Heparinat $11,7 \mu \mathrm{mol} / 1(20 \mathrm{mg} / 100 \mathrm{ml})$, Na-Fluorid $23,81 \mathrm{mmol} / 1(100 \mathrm{mg} / 100 \mathrm{ml}), \mathrm{Na}-\mathrm{Oxalat} 7,45 \mathrm{mmol} / 1$ $(100 \mathrm{mg} / 100 \mathrm{ml})$ und $\mathrm{Na}-\mathrm{Citrat} 19,37 \mathrm{mmol} / 1(500 \mathrm{mg} /$ $100 \mathrm{ml})$. Ascorbinsäure $5,68 \mathrm{mmol} / 1(100 \mathrm{mg} / 100 \mathrm{ml})$ beeinflußt die Meßergebnisse der UV-Methode, der kinetischen Methode und der AutoAnalyzer-Methode nicht; bei der Farbmethode werden falsch positive Ergebnisse erhalten.

Die Wirkung von Arzneimitteln und ArzneimittelMetaboliten auf die Meßergebnisse wird zur Zeit untersucht. Ergebnisse werden zu gegebener Zeit ebenfalls in dieser Zeitschrift publiziert.

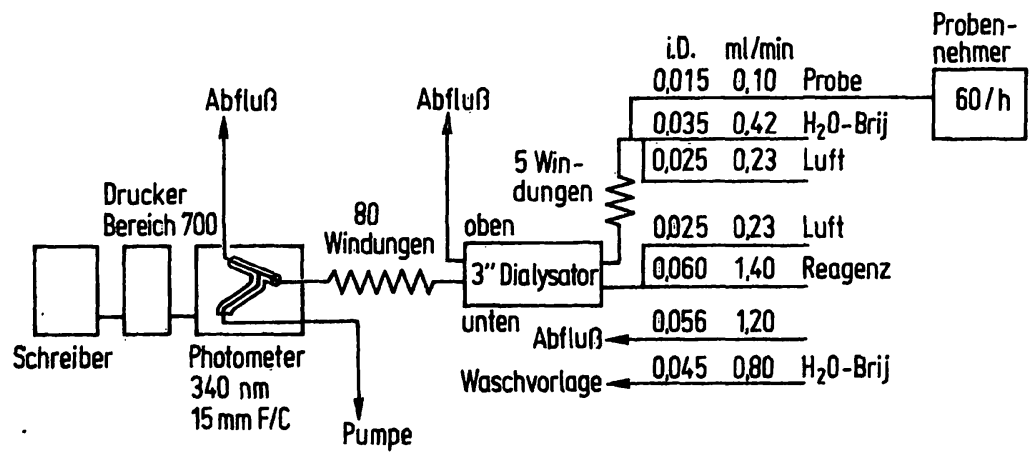

Abb. 3. Glucose-Bestimmung mit Glucose-Dehydrogenase. Fließbild der AutoAnalyzer-II-Methode. Ansatz siehe Text. 
Tab. 4. Glucose-Bestimmung mit Glucose-Dehydrogenase. Vergleich der beschriebenen Methoden-Varianten.

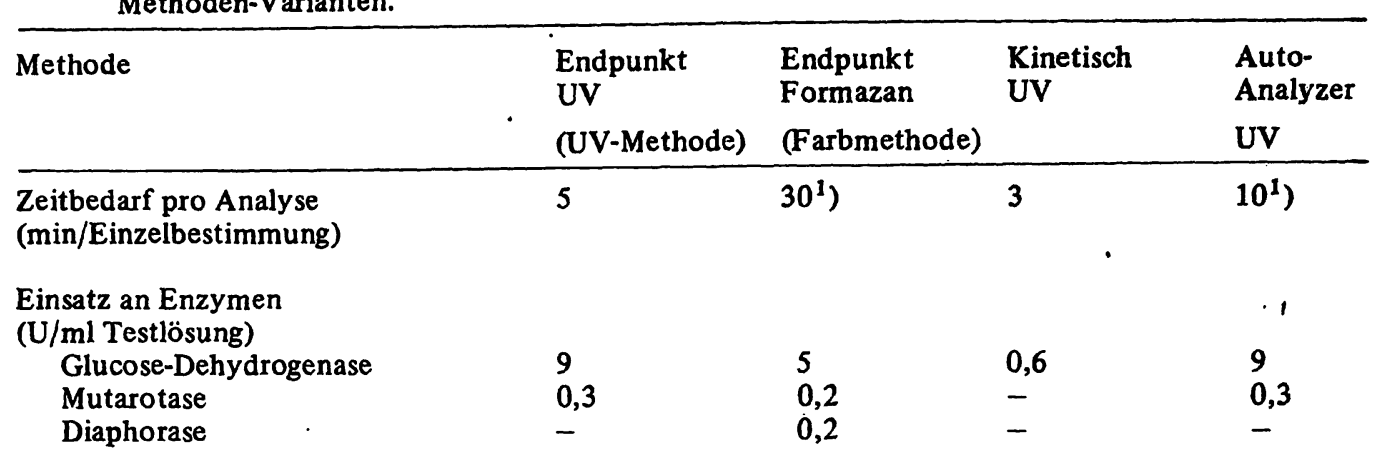

Reproduzierbarkeit

(VK (\%))

$5,55 \mathrm{mmol}$ Glucose/1 Serie (100 mg/100 ml)

$27,75 \mathrm{mmol}$ Glucose/1 Serie $(500 \mathrm{mg} / 100 \mathrm{ml})$

$\begin{array}{lll}5,55 \mathrm{mmol} \mathrm{Glucose} / 1 & & \left.3,6^{2}\right) \\ (100 \mathrm{mg} / 100 \mathrm{ml}) & \mathrm{Tag} / \mathrm{Tag} & \end{array}$

$27,75 \mathrm{mmol} / \mathrm{Glucose} / \mathrm{l}$ $(500 \mathrm{mg} / 100 \mathrm{ml}) \quad \mathrm{Tag} / \mathrm{Tag}$

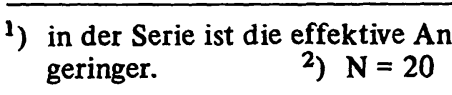

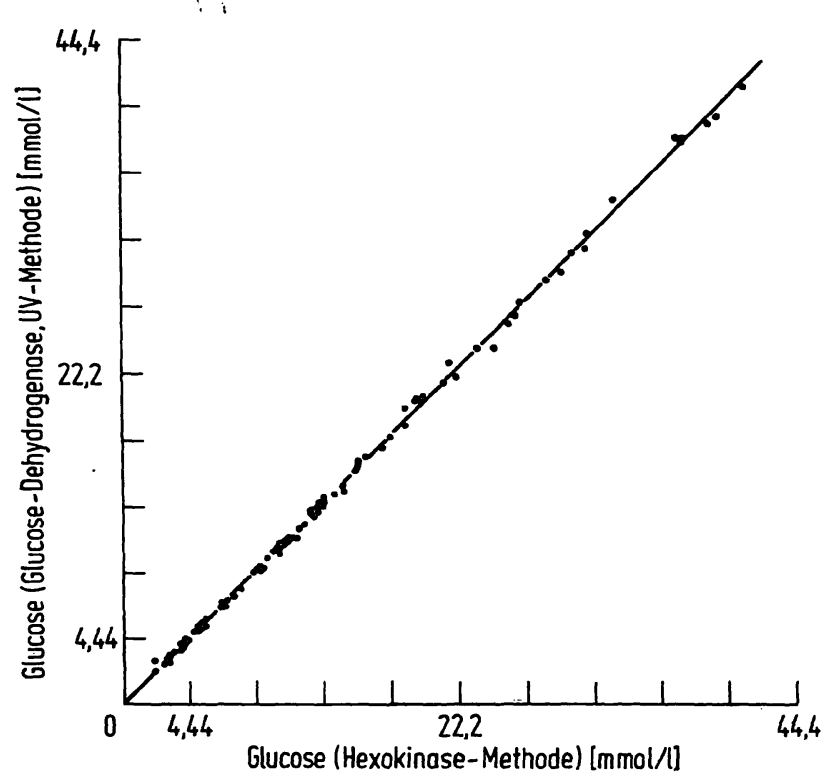

Abb. 4. Glucose-Bestimmung in Humanseren

Vergleich zwischen Hexokinase-Methode (x) und GlucoseDehydrogenase-Methode, Endpunkt-Bestimmung im UV-Bereich (UV-Methode) (y). Ansatz siehe Text. $\mathrm{N}=113 ; \mathrm{y}=-0,217+1,028 \mathrm{x} ; \mathrm{r}=0,999$;

$\bar{x}=13,31 ; \bar{y}=13,48 ; s_{y x}= \pm 0,321$

$95 \%$-Vertrauensbereich von $\mathrm{a}=-0,321$ bis $-0,111$

$95 \%$-Vertrauensbereich von $b=1,022$ bis 1,035

$44,4 \mathrm{mmol} / 1$ Glucose $=800 \mathrm{mg} / 100 \mathrm{ml}$

\section{Methodenvergleich}

Vergleiche gegen die Hexokinase-Methode (23) wurden für die UV-Methode an 113 Humanseren (Abb. 4) und für die AutoAnalyzer-Methode an 176 Humanseren durchgefuhrt (Abb. 5). Die Hexokinase-Methode wurde am AutoAnalyzer entsprechend dem Fließbild von.

Abbildung 3 durchgefuhrt. In beiden Fällen wird bei sehr

$\begin{array}{llll}\left.2,6^{2}\right) & \left.1,7^{2}\right) & \left.2,8^{2}\right) & \left.0,8^{3}\right)\end{array}$

$\left.\left.\left.1,4^{2}\right) \quad 2,6^{2}\right) \quad 2,0^{2}\right) \quad 0,6^{3}$ )

$\begin{array}{llll}\left.3,5^{2}\right) & \left.4,3^{2}\right) & \left.7,6^{2}\right) & \left.0,7^{4}\right)\end{array}$

$\left.1,1^{4}\right)$

$\left.0,7^{4}\right)$ Bestimmung durch versetztes Arbeiten wesentlich
$\begin{array}{ll}\text { 3) } \mathrm{N}=10 & \text { 4) } \mathrm{N}=4\end{array}$

$A \dot{b} b .5$. Glucose-Bestimmung in Humanseren

Vergleich von AutoAnalyzer-II-Methoden (Ansatz siehe Text): Héxokinase-Methode $=x$, Glucose-Dehydrogenase-Methode $=y$.

$N=176 ; y=-0,121+1,004 x ; r=0,998$;

$\overline{\mathrm{x}}=7,98 ; \overline{\mathrm{y}}=7,90 ; \mathrm{s}_{\mathrm{yx}}= \pm 0,188$

$95 \%$-Vertrauensbereich von $\mathrm{a}=-0,189$ bis $-0,049$

$95 \%$-Vertrauensbereich von $\mathrm{b}=0,996$ bis 1,012

$27,75 \mathrm{mmol} / 1$ Glucose $=500 \mathrm{mg} / 100 \mathrm{ml}$

geringer Streubreite gute Ubereinstimmung erzielt; die Referenzmethode zur Uberprüfung der Farbmethode und der kinetischen Methode ist daher die GlucoseDehydrogenase-UV-Methode. Der Vergleich der UVMethode mit der Farbmethode ist in Abbildung 6 und der Vergleich der UV-Methode mit der kinetischen Methode in Abbildung 7 graphisch und statistisch dargestellt. Auch hier stimmen die Ergebnisse gut überein. 


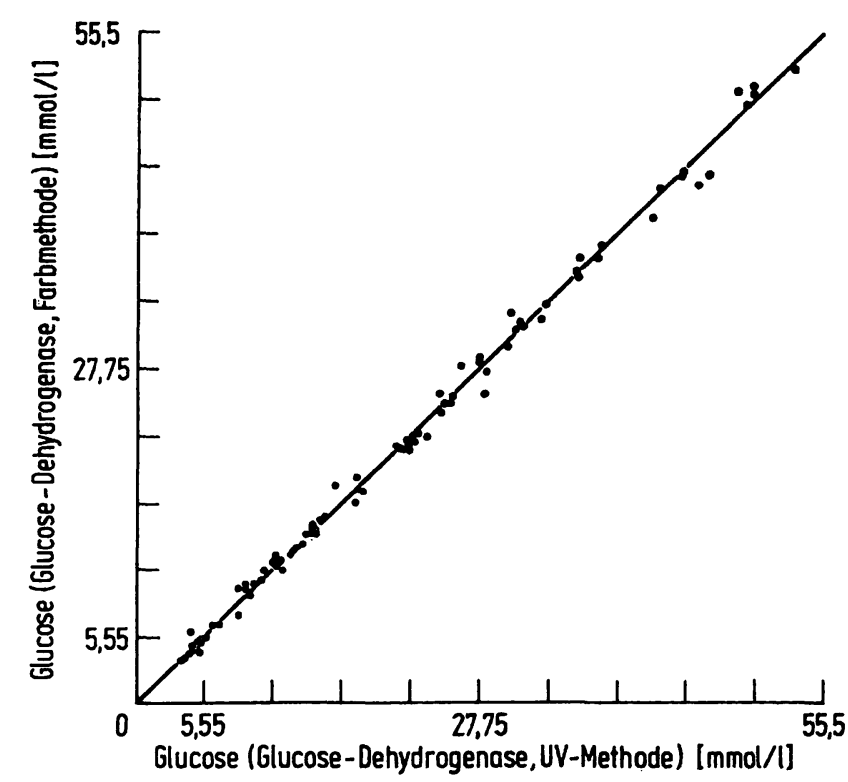

Abb. 6. Glucose-Bestimmung in Humanseren

Vergleich zwischen Varianten der Glucose-Dehydrogenase-Methode: Endpunkt-Bestimmung im UV-Bereich (UV-Methode) (Ansatz siehe Text) $=x$ Bestimmung mit Formazan als Endprodukt (Farbmethode) (Ansatz siehe Text) $=y$

$N=128 ; y=0,066+1,006 x ; r=0,998$

$\bar{x}=16,67 ; \bar{y}=16,84 ; s_{\mathrm{yx}}= \pm 0,721$

$95 \%$-Vertrauensbereich von $a=-0,139$ bis $+0,272$

95\%-Vertrauensbereich von $\mathrm{b}=0,997$ bis 1,016

$55,5 \mathrm{mmol} / 1$ Glucose $=1000 \mathrm{mg} / 100 \mathrm{ml}$

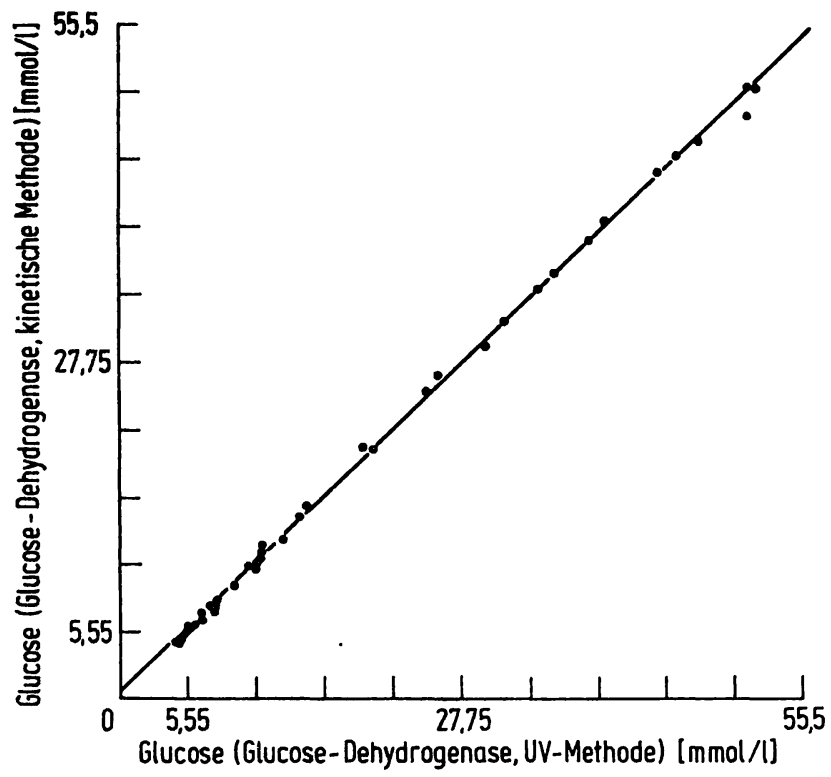

Abb. 7. Glucose-Bestimmung in Humanseren

Vergleich zwischen Varianten der Glucose-Dehydrogenase-Methode: Endpunkt-Bestimmung im UV-Bereich (UV-Methode) (Ansatz siehe Text) $=x$ Kinetische Bestimmung im UV-Bereich (kinetische Methode) (Ansatz siehe Text) $=y$ $N=60 ; y=0,132+0,983 x ; r=0,999$; $\bar{x}=15,21 ; \bar{y}=15,10 ; s_{y x}= \pm 0,461$ $95 \%$-Vertrauensbereich von $\mathrm{a}=-0,038$ bis $+0,305$ $95 \%$-Vertrauensbereich von $b=0,975$ bis 0,992 $55,5 \mathrm{mmol} / 1 \mathrm{Glucose}=1000 \mathrm{mg} / 100 \mathrm{ml}$

\section{Literatur}

1. Metzger, R. P., Wilcox, S. S. \& Wick, A. N. (1964), J. Biol. Chem. 239, 1769-1772.

2. Bach, J. A. \& Sadoff, H. L. (1962), J. Bacteriol. 83, 699 bis 707.

3. Okamura, S., Izaki, K. \& Takahashi, H. (1970), J. Gen. Appl. Microbiol. 16, 429-441.

4. Pauly, W. \& Pfleiderer, G:, Hoppe-Seyler's Z. physiol. Chem., in Vorbereitung.

5. Sadoff, H. L. (1966), in Methods in Enzymology .(Colowick, S. P. \& Kaplan, N. O., ed.), Vol. 9, p. 103-107, Academic Press, New York.

6. Doy, R., Halvorson, H. \& Church, B. (1959), J. Bacteriol. 77, 43-54.

7. Harrison, D. C. (1932), Biochem. J. 26, 1295-1299.

8. Strecker, H. J. \& Korkes; S. (1952), J. Biol. Chem. 196, 769-784.

9. Brink, N. G. (1953), Acta Chem. Scand. 7, 1081-1089.

10. Nakamura, M. (1954), J. Biochem. 41, 67-80.

11. Stary, Z. (1959), Ergebn. Physiol. 50, 174-408.

12. Drube, H.-Ch. (1963), Münch. Med. Wochenschr. 105, 254 bis 259.

13. Dietze, F., Laue, R., Schulz, H.-J. \& Wendtlandt, H. (1973), Deut. Gesundheitsw. 28, 294-301.
14. Bosseckert, H. (1966), Medicamentum 7, 326-329.

15. Hawkins, K. I. (1970), Clin. Chem. 16, 749-752.

16. Ferard, G. \& Metais, P. (1970), Clin. Chim. Acta 30, 279 bis 287.

17. Jolley, R. L., Warren, K. S., Scott, C. D., Jainchill, J. L. \& Freeman, M. L. (1970), Amer. J. Clin. Pathol. 53, 793-802.

18. Gavard, R. \& Combre, C. (1959), C. R. Acad. Sci. 249, 2243 bis 2245 .

19. Bailey, J. M., Fishman, P. H. \& Pentchev, P. G. (1969), J. Biol. Chem. 244, 781-788.

20. Fishman, P. H., Pentchev, P. G. \& Bailey, J. M. (1973), Biochemistry $12,2490-2495$.

21. Bücher, Th., Krell, H., Lusch, G., Graßl, M., Ziegenhorn, J. \& Bergmeyer, H. U. (1974), diese Z. 12, 239-240.

22. Keller, H., Faust, U. \& Becker, J. (1973), Chem. Rundsch. 26, 24-29.

23. Bergmeyer, H. U., Bernt, E., Schmidt, F. \& Stork, H. (1974), in Methoden der enzymatischen Analyse (Bergmeyer, H. U. Hrșg.), Vol. II, p. 1241-1246, Verlag Chemie, Weinheim, 3. Auflage.

Dr. H. Lang Biochemische Forschung E. Merck 61 Darmstadt 2 Postfach 4119 
